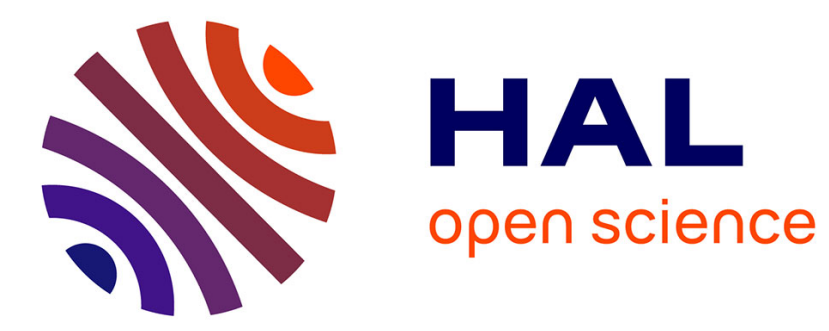

\title{
Imprints of a heliospheric bowshock on interstellar oxygen populations
}

\author{
M. Bzowski, H.-S. Fahr, S. Grzedzielski
}

\section{To cite this version:}

M. Bzowski, H.-S. Fahr, S. Grzedzielski. Imprints of a heliospheric bowshock on interstellar oxygen populations. Astrophysics and Space Sciences Transactions, 2006, 2 (1), pp.1-10. hal-00297474

HAL Id: hal-00297474

https://hal.science/hal-00297474

Submitted on 27 Jan 2006

HAL is a multi-disciplinary open access archive for the deposit and dissemination of scientific research documents, whether they are published or not. The documents may come from teaching and research institutions in France or abroad, or from public or private research centers.
L'archive ouverte pluridisciplinaire HAL, est destinée au dépôt et à la diffusion de documents scientifiques de niveau recherche, publiés ou non, émanant des établissements d'enseignement et de recherche français ou étrangers, des laboratoires publics ou privés. 


\title{
Imprints of a heliospheric bowshock on interstellar oxygen populations
}

\author{
M. Bzowski ${ }^{1}$, H.-J. Fahr ${ }^{2}$, and S. Grzedzielski ${ }^{1}$ \\ ${ }^{1}$ Space Research Centre PAS, Bartycka 18A, 00-716 Warsaw, Poland \\ ${ }^{2}$ Institut für Astrophysik, Universität Bonn, Auf dem Hügel 71, Bonn, Germany
}

Received: 9. August 2005 - Revised: 1 November 2005 - Accepted: 14 December 2005 - Published: 27 January 2006

\begin{abstract}
It is well known that the nearby interstellar medium represents a partially ionized gas composed by different chemical species, amongst them hydrogen, helium and oxygen as the most abundant elements. While the passage of the interstellar protons and $\mathrm{H}$-atoms over the solar system has been satisfactorily well modelled meanwhile, the entrance into the heliosphere of the other interstellar chemical species needs some additional care. Here we especially follow with Boltzmann-kinetic treatments the phase-space history of interstellar oxygen ions and atoms at their passage over the outer heliospheric bowshock and at their further approach towards the inner heliosphere. We describe the nose region of the bowshock as a mild MHD shock of a nearly perpendicular type. While the $\mathrm{O}$-atoms pass over this shock with no velocity-space imprints, by contrast the $\mathrm{O}^{+}$-ions run over the shock structure with a typical overshoot velocity and on the downstream region are then picked up by the frozen-in magnetic fields that comove with the plasma bulk flow, i.e. the proton bulk flow. This leads to a strongly non-relaxated O-ion velocity distribution which substantially differs both from the local O-atom and proton distribution functions. Due to a strong charge exchange coupling between $\mathrm{O}$-ions/atoms and $\mathrm{H}$-atoms/ions the distributions downstream from the bowshock undergo permanent changes which we estimate in this study here. Even though relaxation processes operate by Coulomb collisions, wave-particle interactions and elastic atom-atom collisions, the bowshock imprints on the O- ion and atoms distribution functions may be conserved throughout the whole heliospheric interface, and, as we are going to suggest in this paper, in fact as such may be used as tracers to the physics and the nature of the bowshock. One of the main conclusions is that the population of O-atoms created in the vicinity of the bow-shock should carry a signature of the effective shock strength that could be detectable if the LIC magnetic field is about 2 microgauss or stronger.
\end{abstract}

Correspondence to: M. Bzowski (bzowski@ cbk.waw.pl)

\section{Introduction}

The problem of the heliospheric interface, where $\mathrm{H}$-atoms and protons are effectively coupled by charge exchange interactions, has already often been faced in the literature. Especially it was recognized already since about a decade ago that the passage of neutral interstellar hydrogen and oxygen atoms $(\mathrm{O}, \mathrm{H})$ through the plasma interface ahead of the solar wind termination shock is strongly influenced by the effective charge-exchange coupling of these species to the local plasma structures like those of the inner and the outer heliosheath. A detailed study of these coupling effects needs a kinetic treatment, since the relevant charge exchange mean free paths between $\mathrm{H} / \mathrm{O}$-atoms and protons in the heliosheath are comparable to or even larger than the typical structure scales of the interface plasma flow (i.e. Knudsen numbers are small compared to 1; see Ripken and Fahr, 1983; Fahr and Ripken, 1984; Fahr, 1991; Osterbart and Fahr, 1992; Baranov and Malama, 1993; Fahr et al., 1993; McNutt et al., 1998; Bzowski et al., 2000; Izmodenov et al., 1997). Nevertheless, due to substantial mathematical complications connected with such kinetic treatments of the problem, meanwhile many heliospheric modelings have appeared in the literature which use hydrodynamic treatments of at least two interacting fluids, namely $\mathrm{H}$-atoms and protons, coupled by resonant charge exchange reactions (for recent reviews, see Zank, 1999; Fahr, 2003). The hydrodynamics applied in these approaches produces a description of the space-time behavior of the lowest hydrodynamic moments like density, bulk velocity, and pressure of protons and $\mathrm{H}$-atoms. The local distribution functions are taken as functions of these moments in the form of shifted Maxwellians.

As suggested in the illustrative Fig. 1, the permanent mutual filling of freshly charge-exchanged particles into the local distribution functions of protons and $\mathrm{H}$-atoms will permanently drive the resulting distribution functions away from a highly relaxated three-moment hydrodynamic (HD) distribution, as was investigated in detail by Fahr and Bzowski (2004a,b). This fact has been recog- 
nized and respected for quite some time by Baranov and Malama (1993,1995), who for this reason have developed a kinetic treatment at least for the neutral $\mathrm{H}$-atoms, even in view of the mathematical expenditures which result from such an ambitious approach (Malama, 1991). But to clearly perceive the actual interface physics even the semi-kinetic approach offered by them, where the $\mathrm{H}$-atoms are treated with a kinetic Monte Carlo code, still is based on the assumption that at least the protons can be described as a hydrodynamic fluid. But even this is not true, as is demonstrated by Fahr and Bzowski (2004a,b), since even the protons develop deviations from a 3-moment HD-distribution. Therefore to honestly respect the true situation of two fluids interacting by charge exchange reactions one would need a fully kinetic description both for the $\mathrm{H}$-atoms and the protons. This is especially true in the case when interstellar $\mathrm{O}^{+}$-ions and $\mathrm{O}$-atoms are involved in the interaction, as we are going to show in the following. This kind of highly complicated, fully kinetic modeling is not offered in this paper, but we present calculations for a partially ionized H/O-plasma which clearly manifest the resulting deviations of $\mathrm{H} / \mathrm{O}$-atom and -ion distributions from 3-moment HD distributions. Especially in this paper here we are interested in calculating the $\mathrm{O}^{+}$-ion distribution function which, together with the distribution function of the O-atoms, may serve as ideal tracer of the interface physics.

\section{General interaction concept and theoretical approach}

Here we start from the assumption that proton and hydrogen atoms in the interface play the main role at establishing the plasma structures in the heliospheric interface. In the first order thus we describe the interface by a hydrodynamic twofluid (i.e. H-atom and proton) interaction model developed by Fahr et al. (2000) and specifically used in the paper by Fahr and Bzowski (2004a). From this model calculations we obtain the hydrodynamic $\mathrm{H}$-atom and $\mathrm{H}^{+}$-ion moments as functions of space coordinates. $\mathrm{O}$-atoms and $\mathrm{O}^{+}$-ions, which are also transported towards the solar system by the interstellar medium flow, are considered here as minor constituents which can be treated as co-moving test populations since they contribute negligible fractions to the mass-, momentum- and energy-densities of the multifluid mixture.

The reason for this assumption is given by the fact that the cosmic O-to-H abundance is estimated as $\alpha(O / H)=\left[n_{O^{+}}+n_{O}\right] /\left[n_{H^{+}}+n_{H}\right]=(3.45-6.8) \cdot 10^{-4}$ (see, respectively, Oliveira et al., 2005 and Cameron, 1973). In addition, in the unperturbed interstellar medium one can rely on a charge exchange equilibrium of closely coupled $\mathrm{O}$ - and $\mathrm{H}$-species yielding the fact that $\left[n_{O^{+}} / n_{O}\right]=(8 / 9)\left[n_{H^{+}} / n_{H}\right]$ (see, e.g. Stebbings et al., 1964). Introducing the ionization degrees $\xi_{H, O}$ we thus find that the relation $\left[\xi_{O} /\left(1-\xi_{O}\right)\right]=(8 / 9) \cdot\left[\xi_{H} /\left(1-\xi_{H}\right)\right]$ must be valid. Assuming furthermore that the LISM hydrogen is ionized by $\sim 50$ percent (i.e. $\xi_{H}=0.5$ ), hence permits to conclude from the above that $\xi_{\mathrm{O}}=0.47$. Now comparing
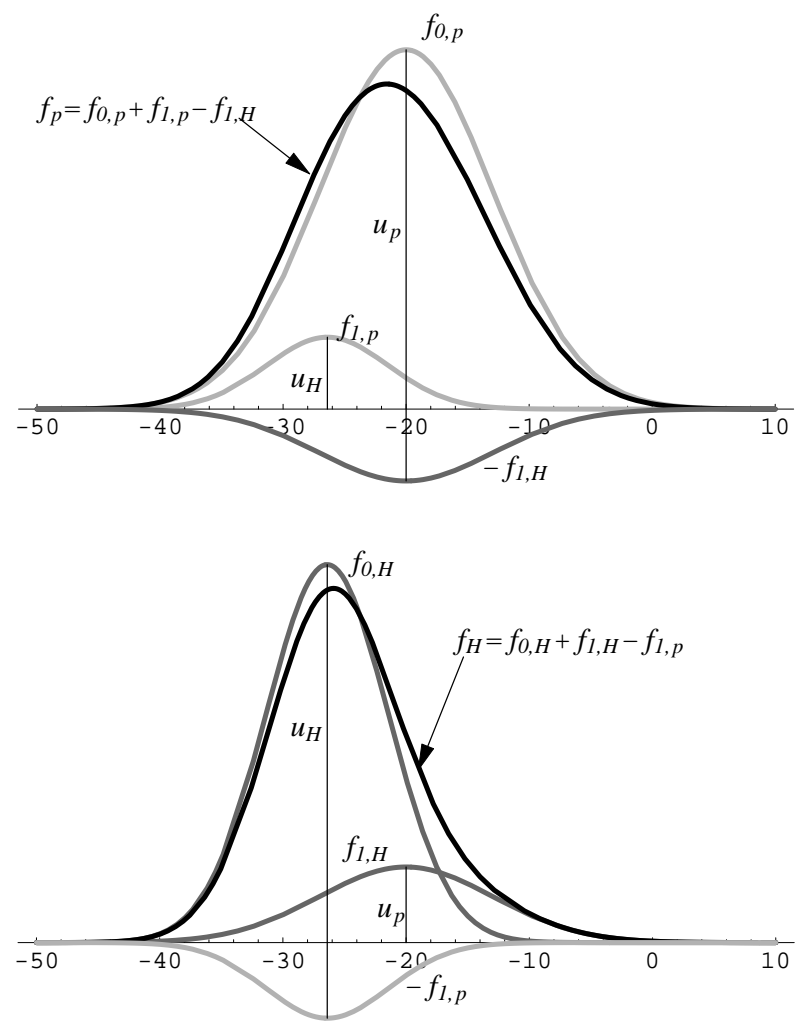

Fig. 1. Illustration of plasma - neutral gas interaction within the interface. Cuts through the velocity distribution functions of $\mathrm{H}-$ atoms (lower plot) and protons (upper plot) are shown at a location on the stagnation line within the outer heliosheath. $f_{0, p / H}$ are the unperturbed hydrodynamic distribution functions of protons and $\mathrm{H}$-atoms, respectively, while $f_{1, p / H}$ are the deviations of the respective distribution functions due to charge exchange. $f_{p / H}$ are the resulting (modified) distribution functions (after Fahr and Bzowski, 2004a).

the mass densities of the $\mathrm{O}^{+}$-ions with that of the protons one obtains:

$$
\begin{aligned}
\rho_{O^{+}} / \rho_{p} & =\frac{m_{O} \xi_{O}\left[n_{O^{+}}+n_{O}\right]}{m_{p} \xi_{H}\left[n_{p}+n_{H}\right]} \\
& =16 \cdot \frac{0.47}{0.5} \cdot 6.8 \cdot 10^{-4}=10^{-2}
\end{aligned}
$$

meaning that only 1 percent of the mass density of the LISM plasma is represented by $\mathrm{O}^{+}$-ions, in other words that the predominant mass of this plasma is represented by the $\mathrm{H}^{+}$-ions.

We therefore adopt that the plasma dynamics in the interface is determined as given in the solutions presented by Fahr and Bzowski (2004a), i.e. as if no $\mathrm{O}^{+}$-ions had been present. In the unperturbed LISM region, far ahead of the solar system, thermodynamic equilibrium between $\mathrm{O}^{+}$- and $\mathrm{H}^{+}$-ions will be established. Therefore the distribution function of $\mathrm{O}^{+}$-ions will be a Maxwellian shifted by the upstream plasma bulk velocity $U_{1}$ with a temperature equal to that of the protons, i.e. here one has: $T_{O 1}=T_{H 1}=T_{1}$.

At the outer bow shock the plasma bulk velocity is reduced from its upstream value $U_{1}$ to its downstream value $U_{2}$. To 
physically arrange for that deceleration of the proton plasma, an electric potential wall $\Delta \Phi$ must be established. However fluctuating in magnitude due to electrostatic oscillations it may be over short periods, in a time average over longer periods it will have the following magnitude:

$e \Delta \Phi \simeq \frac{1}{2} m_{p}\left(U_{1}^{2}-U_{2}^{2}\right)$

The $\mathrm{O}^{+}$-ions immediately after passing over this bow shock may thus have a kinetic overshoot velocity $\boldsymbol{U}_{O, 2}$ with respect to the downstream plasma bulk frame. For a quasiperpendicular shock this velocity will be parallel to $\boldsymbol{U}_{2}$ and $\boldsymbol{U}_{1}$ and is given by:

$U_{O, 2}^{2}=U_{1}^{2}-\left(m_{p} / m_{O}\right)\left[U_{1}^{2}-U_{2}^{2}\right]=\frac{15}{16} U_{1}^{2}+\frac{1}{16} U_{2}^{2}$

Since the downstream plasma flow is controlled by the protons moving with a bulk velocity $U_{2}$ and since the background magnetic field essentially is frozen into this flow, the differential overshoot velocity $\left|\left(\boldsymbol{U}_{O, 2}-\boldsymbol{U}_{2}\right) \times \frac{B}{B}\right|=\Delta U$ of the $\mathrm{O}^{+}$-ions (which is of the order of the velocity difference between the upstream and downstream sides) is converted into gyrational motions of these ions perpendicular to the field direction of the convected magnetic field.

\section{Structure of the hydromagnetic bow shock}

Protons and $\mathrm{O}^{+}$ions will be coupled most strongly to neutral hydrogen by charge exchange. For average relative speeds between these particles of $25 \mathrm{~km} / \mathrm{s}$ and atom densities of $n_{H I}=0.1 \mathrm{~cm}^{-3}$, the neutralization times by charge exchange are $10^{9}, 2 \cdot 10^{14}$ and $10^{10} \mathrm{~s}$, respectively, for the three mentioned ion species (these estimates are based on rates/cross sections as given by Maher and Tinsley, 1977; Kingdon and Ferland, 1996; Chambaud et al., 1980). Therefore, for a time of $\tau \leq \sim 10^{8}-\sim 10^{9}$ s plasma that experienced the bowshock transition may be treated as dynamically decoupled from the neutrals. As we show below, for plausible magnetic field strengths, the downstream plasma velocity in the (stationary) shock frame will be from $\sim 10$ to $26 \mathrm{~km} / \mathrm{s}$, depending on the effective bowshock compression ratio (the highest velocity meaning there is no shock at all). Therefore the thickness $D$ of the shocked plasma layer with velocity and temperature different from interstellar values could be of the order of $D \simeq\left(10 \mathrm{~km} / \mathrm{s} \cdot 10^{8-9} \mathrm{~s}\right)=10^{14-15} \mathrm{~cm}$ which yields about one (or more) tens of AU. The question now arises, whether or not this plasma can produce particle population signatures detectable in the inner heliosphere? Should the answer be positive, one might get a way to learn something on the details of the bowshock physics and possibly on the upstream magnetic field $B_{1}$.

The most reliable data on the local interstellar medium in front of the heliosheath and the bowshock (Witte et al., 2004; Witte, 2004) indicate (assuming a time-independent situation in the solar frame), that the upstream sonic Mach number $M_{s 1}=U_{1} / c_{s 1}>1$ is known fairly accurately, while the corresponding magnetosonic number $M_{m s, 1}$ is not. Here $c_{s 1}$ denotes the ordinary sound speed of upstream plasma. For a plasma mixture composed of protons, $\mathrm{He}^{+}$-ions and electrons, $M_{s 1}$ is about 2, with some weak dependence on the not-so-well known ionization degrees of hydrogen and helium. It is therefore convenient to look for bowshock properties for varying magnetic field strengths, assuming $M_{s 1}$ to be known.

Here we adopt the simplest approach, considering a singlefluid, hydromagnetic, perpendicular shock which, strictly speaking, may only apply, if at all, to the bowshock nose. The shock compression ratio $s=\rho_{2} / \rho_{1}=U_{1} / U_{2}=B_{2} / B_{1}$ is given in terms of $M_{s 1}$ and the upstream plasma-beta $\beta_{1}$ by the equation (cf. Diver, 2001)

$$
\begin{aligned}
2(2-\gamma) s^{2}+\left\{2+\beta_{1}\right. & {\left.\left[2+(\gamma-1) M_{s 1}^{2}\right]\right\} } \\
\cdot & \gamma s-\gamma(\gamma+1) M_{s 1}^{2} \beta_{1}=0
\end{aligned}
$$

and thus the ratio of the temperatures on the two sides of the shock can be written as

$T_{2} / T_{1}=\left[1+\gamma M_{s 1}^{2}(1-1 / s)+\left(1-s^{2}\right) / \beta_{1}\right] / s$

where indices 1,2 refer to upstream, downstream values; $\gamma$ is the adiabatic exponent; velocities are defined in the shock frame; $\rho_{1}$ and $\rho_{2}$ denote the plasma mass densities; and $\beta_{1}=2 c_{s 1}^{2} / \gamma c_{A 1}^{2}$, where $c_{A 1}$ is the unknown upstream Alfvén velocity.

The effect of the unknown interstellar magnetic field on downstream plasma properties is shown in Fig. 2 (upper panel). The case shown corresponds to a neutral hydrogen density in the Local Interstellar Cloud (LIC) of $n_{H_{1}}=0.18$ atom $/ \mathrm{cm}^{3}$, a temperature of $T_{1}=6300 \mathrm{~K}$, an elemental abundance by number of $[\mathrm{H}] /[\mathrm{He}]=10$, and a polytropic index of $\gamma=5 / 3$. This set of parameters is called LIC1. To illustrate the influence upon the subshock of the possible uncertainties in these parameters another set of values (called LIC2) is used in the lower panel of Fig. 2.

1: LIC1 $-\mathrm{H}$ ionization: $25 \%$; LIC $-\mathrm{He}$ ionization: $37.5 \%$, yielding $n_{1}(\mathrm{e})=0.069 \mathrm{~cm}^{-3}, n_{1}(\mathrm{p})=0.06 \mathrm{~cm}^{-3}$, $n_{1}(\mathrm{HeII})=0.009 \mathrm{~cm}^{-3}, T=6300 \mathrm{~K}, v_{\mathrm{LIC}}=26.4 \mathrm{~km} / \mathrm{s}$;

2: LIC2 - H ionization: 50\%; LIC - He ionization: $50 \%$, yielding $n_{1}(\mathrm{e})=0.11 \mathrm{~cm}^{-3}, n_{1}(\mathrm{p})=0.1 \mathrm{~cm}^{-3}$, $n_{1}(\mathrm{HeII})=0.01 \mathrm{~cm}^{-3}, T=8000 \mathrm{~K}, v_{\mathrm{LIC}}=26.4 \mathrm{~km} / \mathrm{s}$.

It is evident that the (plasma) bowshock may be relatively strong $(s \sim 2.5)$ only for very weak fields, i.e. $B_{1} \leq 1$ microgauss. For recently discussed values of the local interstellar magnetic fields with values of $2-3$ microgauss (Cox and Helenius, 2003; Frisch, 2004) the bowshock either is very weak or completely disappears. However, in the case when a reasonably robust $(s \sim 2)$ bowshock does exist, the downstream population of $\mathrm{O}^{+}$-ions may differ from both the original interstellar $\mathrm{O}^{+}$population and the $\mathrm{O}^{+}$population which would simply result from the thermal heating of interstellar plasma by the shock-induced slowdown of the plasma bulk flow in the vicinity of the heliopause. If in this layer enough of relatively fast neutral $\mathrm{O}$-atoms could be produced out of $\mathrm{O}^{+}$by charge exchange with $\mathrm{H}$-atoms, then an imprint of the bowshock could be present in the $\mathrm{O}$-atom velocity distribution in 

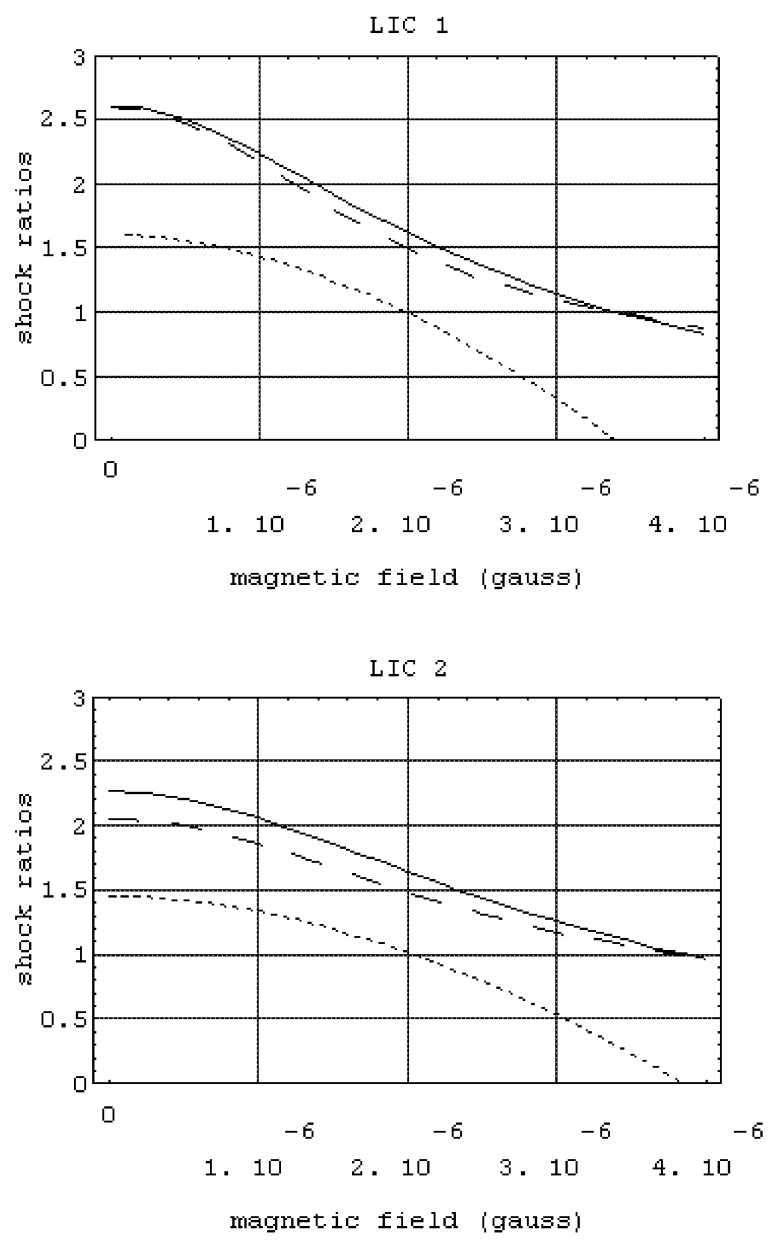

Fig. 2. Shown as functions of the upstream interstellar magnetic field $B_{1}$ in gauss are: bowshock compression $s=\rho_{2} / \rho_{1}$ (solid lines); temperature ratio $T_{2} / T_{1}$ (dashed lines); velocity difference $\left(U_{1}-U_{2}\right) / 10 \mathrm{~km} / \mathrm{s}$ (dotted lines). The upper panel corresponds to LIC1: $\mathrm{H}$ ionization $25 \%$; He ionization $37.5 \%$, yielding $n(\mathrm{e})=0.069 \mathrm{~cm}^{-3}, n(\mathrm{p})=0.06 \mathrm{~cm}^{-3}$, $n(\mathrm{HeII})=0.009 \mathrm{~cm}^{-3}, T=6300 \mathrm{~K}, v_{\mathrm{LIC}}=26.4 \mathrm{~km} / \mathrm{s}$. The lower panel corresponds to LIC2: H ionization 50\%; He ionization 50\%, yielding $n(\mathrm{e})=0.11, n(\mathrm{p})=0.1 \mathrm{~cm}^{-3}, \quad n(\mathrm{HeII})=0.01 \mathrm{~cm}^{-3}$, $T=8000 \mathrm{~K}, v_{\mathrm{LIC}}=26.4 \mathrm{~km} / \mathrm{s}$. Note that for $B_{1}$ strong enough the shock solutions become unphysical (compression $s<1$, velocity difference $U_{1}-U_{2}<0$ ). This corresponds to situation when the magnetosonic speed exceeds the LIC plasma speed.

the inner heliosphere that might be detectable in the studies of $\mathrm{O}^{+}$pickup ion distributions.

The problem we are facing here is how wide could be the post-shock layer in which the $\mathrm{O}^{+}$-ions may remain not yet thermalized, but carrying clear imprints from the shock passage? The unperturbed velocity distribution of $\mathrm{O}$-atoms and -ions upstream of the bowshock in the LIC may be adopted as an equilibrium distribution with $T_{1}=6300 \mathrm{~K}$ (for LIC1). The r.m.s. thermal velocity of the O-atoms then is $3.1 \mathrm{~km} / \mathrm{s}$. For $B_{1}=1$ microgauss and $s=2$, the velocity difference $U_{1}-U_{2}$ across the shock is $16(14) \mathrm{km} / \mathrm{s}$ for LIC1 (LIC2). The $\mathrm{O}^{+}$-ions will thus enter the shocked plasma with an overshoot velocity of that magnitude, i.e. $\mathrm{O}^{+}$in the core of the distribution will have energies $15-20 \mathrm{eV}$ per ion. In velocity space the ions will form a ring - or rather a torus. Removal of $\mathrm{O}^{+}$-ions from the torus may occur by charge exchange with neutral hydrogen, by Coulomb scattering on protons, by inelastic collisions with neutral species, and (possibly) by scattering on waves.

The time for charge exchange is $10^{10} \mathrm{~s}$, as mentioned earlier. Coulomb scattering will (1) decrease the torus diameter in velocity space and (2) increase the torus width. The first effect is faster and gives an e-folding loss time scale of $10^{8} \mathrm{~s}$, the second will be an order of magnitude slower. Also of inferior importance will be scattering by inelastic collisions. Therefore, unless the $\mathrm{O}^{+}$-ions resonate with other plasma processes (like MHD waves) transmitted or engendered by the shock, the torus distribution may survive for about 3 years. For $s=2$ this translates into a width of 5 $10 \mathrm{AU}$, which does not exceed the evaluation of the width of shocked plasma decoupled dynamically from the flow of neutrals. The conclusion of these simple estimates is that indeed, behind a bowshock, a plasma layer could be developed, extending over a rather small fraction of the total distance from the bowshock nose to the heliopause, and containing $\mathrm{O}^{+}$-ions of energies distinctly above the thermal energies of the main plasma and gas constituents.

\section{Bow shock physics: two possible scenarios}

A bowshock may precede the heliopause if the magnetosonic Mach number for the plasma flow in the local interstellar medium relative to the solar system is large enough (i.e. $M_{m s}>1$ for perpendicular shocks). In view of very little direct observational evidence being available on the upstream local interstellar magnetic field $B_{1}$ we shall discuss briefly here conditions for shock formation assuming for simplicity the field 'at infinity' as perpendicular to the flow, with a magnitude not exceeding a few microgauss (Frisch, 2003).

In general, the shock transition may involve both plasma and neutral populations. However, the approaching interstellar plasma - being a fluid with an effective mean free path on the order of Larmor radius - may feel the presence of the heliospheric obstacle much sooner, more effective and more abrupt than neutral populations with their very large mean free paths due to atom-atom and charge exchange collisions. One can thus presume that a plasma subshock (bow shock) will develop, separate from the general transition, in which dynamic conditions prevail with no equilibrium between ions and atoms.

In the following, we present two scenarios of the passage of oxygen ions through the heliospheric bow shock:

(a) We assume that the magnetic field change at the bow shock is so abrupt that ions cannot adiabatically adapt to the increase in the B-field magnitude.

(b) We assume that the transition from the upstream to downstream magnetic field is occurring over a distance $L$ which is large with respect to the ion gyroradius. Then the 
ions have to conserve their magnetic moment at their passage through the bow shock.

We present resulting distribution functions of the $\mathrm{O}^{+}$-ions and discuss their signatures transmitted to the population of O-atoms that could survive the travel through the heliospheric interface to the heliopause.

\subsection{Abrupt MHD shock: Non-conservation of the magnetic moment}

The changes in the velocity distribution function of the interstellar $\mathrm{O}^{+}$-ions as they cross the heliosphere bowshock are expected to depend on the detailed (and so far completely unknown) space- and time-structures of this interstellar shock transition. Some guidance as to the physics of these processes could be taken from the existing body of experimental data taken at the Earth bowshock by the CLUSTER spacecraft instrumentation. These data indicate that the efficiency of ion scattering, and thus reflection at quasi-perpendicular shocks, depends strongly on the shock Mach number. Reflection is known to depend on the first critical Mach number $M_{c}$. For shocks with Alfvén Mach numbers above $M_{c}$ reflection is necessary to have dissipation. As both the magnetosonic and (ordinary) sonic Mach numbers for heliosphere bowshock are probably relatively low $(\sim 1.5 / \sim 2$ for the former/latter, depending on interstellar magnetic field strength), one may presume the shock is sub-critical and ion scattering and reflection are not the dominant processes determining the downstream velocity distribution function for the $\mathrm{O}^{+}$-ions. Instead, we assume that the 'heating' of heavy ions as they enter the downstream region is related to their non-deflection at the shock ramp (Lee et al., 1986). This requires that the shock ramp thickness, assumed to be of the order of the ion inertial length $c / \omega_{p i}$, is much lower than the Larmor radius $\left(v_{i} m_{O} c\right) /(e B)$. Here $\omega_{p i}=\sqrt{4 \pi e^{2} n / m_{p}}$ is the ion plasma frequency of the dominant ion species (protons), $m_{O}$ is the $\mathrm{O}^{+}$-ion mass, $m_{p}$ is the proton mass, $n$ the plasma density, $B$ magnetic field intensity and $v_{i}$ is a typical upstream velocity of $\mathrm{O}^{+}$-ions. The condition mentioned becomes then

$v_{i} \gg c_{A} m_{p} / m_{O}$

(where $c_{A}$ denotes the Alfvén speed), which seems to be satisfied for $B$-field intensities small enough to secure a shock transition. The approach described was also recently used to explain shock heating at solar shocks observed by UVCS/SOHO (Mancuso et al., 2002).

In the following we assume the heliosphere bowshock is perpendicular (with normal to the shock along the $z$-axis) and the velocity distribution function of $\mathrm{O}^{+}$-ions on the upstream side is isotropic in the frame of bulk interstellar plasma, i.e. a Gaussian in each Cartesian velocity coordinate

$d N\left(v_{j}\right)=N /(a \sqrt{\pi}) \exp \left[-\left(v_{j} / a\right)^{2}\right] d v_{j}$

where $a$, the most probable speed, is given by $a^{2}=2 K T_{1} / m_{O}$ and $T_{1}$ is equal to the observed temperature of the LIC.
At the shock transition the velocity component $v_{\|}$parallel to the magnetic field (assumed to be oriented along the $x$-axis) stays unchanged and is decoupled from the other ones. However, $v_{z}$ and $v_{y}$ are constantly coupled by cyclotron gyration and under present assumptions a relationship of the form $v_{\perp}^{2}=\left(w+v_{z}\right)^{2}+v_{y}^{2}$ has to be satisfied immediately past the shock downstream, where $w=U_{1}-U_{2}$ is the velocity difference between bulk upstream and downstream plasma speeds and $v_{\perp}$ is the $\mathrm{O}^{+}$-ion post-shock perpendicular velocity.

In this way instead of an upstream 2D probability distribution function (PDF) of two random variables $v_{z}, v_{y}$ :

$$
\begin{aligned}
f_{z y}\left(v_{z}, v_{y}\right) d v_{z} d v_{y} & =A \exp \left[-\left(v_{z} / a\right)^{2}\right] \\
& \cdot \exp \left[-\left(v_{y} / a\right)^{2}\right] d v_{z} d v_{y}
\end{aligned}
$$

one has to deal with a 1-D PDF (called $f_{\perp}\left(v_{\perp}\right)$ ) of a new random variable $v_{\perp}$. Using standard transformations for calculating a PDF of a random variable being a function of two other random variables one gets

$$
\begin{gathered}
f_{\perp}\left(v_{\perp}\right)=A \exp \left[-(w / a)^{2}\right] v_{\perp} \exp \left[-\left(v_{\perp} / a\right)^{2}\right] \\
\cdot \int\left(v_{\perp}^{2}-v_{y}^{2}\right)^{-1 / 2} \exp \left[2 w\left(v_{\perp}^{2}-v_{y}^{2}\right)^{1 / 2} / a^{2}\right] d v_{y}
\end{gathered}
$$

The spread of velocities in the downstream plasma frame of ions going at any moment sunwards or in the opposite directions is determined by the spread of, appropriately, $-v_{\perp}$-values and $+v_{\perp}$-values as determined by the $f_{\perp}$ distribution function. To get the velocity distribution function of the downstream $\mathrm{O}^{+}$-ions in the solar frame one has to shift the distribution by $-U_{2}$ in velocity space representing the bulk speed of downstream plasma in the solar frame.

\subsection{Mild MHD shock: magnetic moment conservation}

Here we assume that $\mathrm{O}^{+} / \mathrm{O}$ particles are latently involved in the shock physics, i.e. that due to their very small contributions to the momentum and energy densities of the bulk plasma they do not directly interfere with the shock transition of the thermodynamic properties. Hence, essentially an HD-shock is established, similar to that one used to describe the interface plasma flow in Fahr and Bzowski (2004a), in which - in addition to protons - minor species populations like $\mathrm{O}$-atoms and -ions can be treated as test populations. We thus start here from the given knowledge of the downstream properties of LISM $\mathrm{H}^{+}$-ions and $\mathrm{H}$-atoms, but now we would like to describe the change of the upstream $\mathrm{O}^{+}$-ion distribution function into the associated downstream one. We hereby follow essentially the argumentation used by Fahr and Lay (2000) to describe the pick-up ion passage over the solar wind termination shock. To describe the conversion into the downstream $\mathrm{O}^{+}$-distribution function three requirements have to be fulfilled:

1. We assume that downstream of the shock due to the action of frozen-in magnetic fields the two plasma constituents, i.e. $\mathrm{H}^{+}$- and $\mathrm{O}^{+}$-ions, co-move with identical bulk velocities 
$U_{2, H^{+}}=U_{2, O^{+}}=U_{2}$, whose values are adopted as negative. Then, adopting that no $\mathrm{O}^{+}$-ions are reflected at the shock, flux conservation requires that:

$\frac{U_{1}}{U_{2}}=\frac{\rho_{2, p}}{\rho_{1, p}}=\frac{n_{2, O^{+}}}{n_{1, O^{+}}}=s$

2. Assuming furthermore that some small magnetic field is frozen into the plasma flow, then at quasi-perpendicular MHD shock conditions this leads to an increase in the magnetic field magnitude given by $s \simeq s_{B}=B_{2} / B_{1}$. If oxygen ions at their passage over the shock have to conserve their magnetic moments (i.e. if $r_{g O} \leq L$, $r_{g O}=\left[m_{O} c v_{\perp O} /(e B)\right]_{1}$ being the $\mathrm{O}^{+}$-ion gyroradius, and $L$ being the shock transition scale), one then - without the overshoot velocity taken into account - would find that $\left[v_{\perp O}^{2} / B\right]_{1}=\left[v_{\perp O}^{2} / B\right]_{2}$ and $v_{\| O, 2}=v_{\| O, 1}$, where indices " $\perp$ " and " $\| "$ mean velocity components perpendicular and parallel to the local magnetic field $\boldsymbol{B}$. This would yield the relation between the downstream and upstream velocities in the form:

$v_{\perp O, 2}^{2}=s v_{\perp O, 1}^{2}$

Now, this taken together with the assumption that the kinetic overshooting velocity, derived in Eq. (3), converts into addi- tional gyrational energy of the degrees of freedom perpendicular to the magnetic field, one may then conclude, however, that instead of Eq. (3) rather the following velocity transformation should be valid:

$$
\begin{aligned}
v_{\perp O, 2}^{2} & =s\left(v_{\perp O, 1}-\left(U_{2, O}-U_{2}\right)\right)^{2} \\
v_{\| O, 2} & =v_{\| O, 1}
\end{aligned}
$$

3. Furthermore, the Liouville theorem requires the phase space flux conservation, which for the un-normalized distribution functions $f_{\mathrm{O}^{+}}^{*}$ of the $\mathrm{O}^{+}$-ions, valid in the respective plasma bulk frames and indicated by $*$, can be written in the following form:

$f_{O^{+}, 1}^{*} U_{1} d^{3} v_{1}=f_{O^{+}, 2}^{*} U_{2} d^{3} v_{2}$

which thus allows to derive the following relation:

$$
f_{O^{+}, 2}^{*}=f_{O^{+}, 1}^{*} \frac{U_{1}}{U_{2}} \frac{d^{3} v_{1}}{d^{3} v_{2}}=J_{1,2} s f_{O^{+}, 1}^{*}
$$

where the Jacobian transformation matrix $J_{1,2}$ is given by:

$J_{1,2}=\frac{2 \pi v_{\perp, 1} d v_{\perp, 1} d v_{\|, 1}}{2 \pi v_{\perp, 2} d v_{\perp, 2} d v_{\|, 2}}=\frac{v_{\perp, 1}}{s\left(v_{\perp, 1}-\left(U_{2, O}-U_{2}\right)\right)}$

Thus one obtains:

$f_{O^{+}, 2}^{*}\left(v_{\perp, 2}, v_{\|, 2}\right)=\frac{v_{\perp, 1}}{\left(v_{\perp, 1}-\left(U_{2, O}-U_{2}\right)\right)} f_{O^{+}, 1}^{*}\left(v_{\perp, 1}\left(v_{\perp, 2}\right), v_{\|, 1}\left(v_{\|, 2}\right)\right)$

and, reminding that in the upstream wind frame $f_{O^{+}}^{*}\left(v_{\perp}, v_{\|}\right)$appears as a simple Maxwellian, that
$n_{O^{+}, 1} / n_{O^{+}, 2}=1 / s$ and that $v_{\|, 1}=v_{\|, 2}$, it then leads to the following expression:

$f_{O^{+}, 2}^{*}\left(v_{\perp, 2}, v_{\|, 2}\right)=\frac{v_{\perp, 1} n_{O^{+}, 1}}{\left(v_{\perp, 1}-\left(U_{2, O}-U_{2}\right)\right)}\left(\frac{m_{O}}{2 \pi K T_{1}}\right)^{3 / 2} \exp \left[-\frac{m_{O}}{2 K T_{1}}\left(v_{\|, 2}^{2}+\frac{1}{s}\left(v_{\perp, 2}+\sqrt{s}\left(U_{2, O}-U_{2}\right)\right)^{2}\right)\right]$

or

$$
\begin{aligned}
& f_{O^{+}, 2}^{*}\left(v_{\perp, 2}, v_{\|, 2}\right)=\frac{v_{\perp, 2}+\sqrt{s}\left(U_{2, O}-U_{2}\right)}{v_{\perp, 2}} n_{O^{+}, 1}\left(\frac{m_{O}}{2 \pi K T_{1}}\right)^{3 / 2} \\
& \cdot \exp \left[-\frac{m_{O}}{2 K T_{1}}\left(v_{\|, 2}^{2}+\frac{1}{s}\left(v_{\perp, 2}+\sqrt{s}\left(U_{2, O}-U_{2}\right)\right)^{2}\right)\right]
\end{aligned}
$$

Since in the plasma frame we have the Cartesian velocity components related to $v_{\perp, 2}, v_{\|, 2}$ by:

$v_{z}^{*}=v_{\perp, 2} \cos \phi$

$v_{y}^{*}=v_{\perp, 2} \sin \phi$

$v_{x}^{*}=v_{\|, 2}$

where $v_{z}$-axis is oriented parallel to the stagnation line, $v_{x}$ is parallel to $\boldsymbol{B}$, and $\phi$ is the clock angle of an ion gyrat- ing about the magnetic field line, in the solar frame we thus obtain:

$v_{z}=v_{\perp, 2} \cos \phi+U_{2}$

$v_{y}=v_{\perp, 2} \sin \phi$

$v_{x}=v_{\|, 2}$

and with the $v_{x}, v_{y}, v_{z}$ values specified in Eq. (20), this further yields

$f_{O^{+}, 2}\left(v_{\perp, 2}, v_{\|, 2}, \phi\right)=\frac{\left(v_{z}-U_{2}\right) / \cos \phi+\sqrt{s}\left(U_{2, O}-U_{2}\right)}{\left(v_{z}-U_{2}\right) / \cos \phi} n_{O^{+}, 1}\left(\frac{m_{O}}{2 \pi K T_{1}}\right)^{3 / 2} \exp \left[-\frac{m_{O} w^{2}}{2 K T_{1}}\right]$ 
where

$w^{2}=v_{\|, 2}^{2}+\frac{1}{s}\left(\frac{v_{z}-U_{2}}{\cos \phi}+\sqrt{s}\left(U_{2, O}-U_{2}\right)\right)^{2}$

Since further on we are interested only in a cut through the distribution function along the $v_{z}$-axis, we set $v_{\|, 2}=0$ and $\phi=0$ or $\phi=\pi$ and for our specific purposes we obtain the unnormalized function in the solar rest frame in these coordinates by:

$f_{O^{+}, 2}\left(v_{z}\right)=n_{O^{+}, 1}\left[1+\frac{\sqrt{s}\left(U_{2, O}-U_{2}\right)}{\left(v_{z}-U_{2}\right)}\right]\left(\frac{m_{O}}{2 \pi K T_{1}}\right)^{\frac{3}{2}} \exp \left[-\frac{m_{O}}{2 K s T_{1}}\left(v_{z}-U_{2}+\sqrt{s}\left(U_{2, O}-U_{2}\right)\right)^{2}\right]$

for $\phi=0, v_{z}>U_{2}-\sqrt{s}\left(U_{2, O}-U_{2}\right)$ and

$f_{O^{+}, 2}\left(v_{z}\right)=n_{O^{+}, 1}\left[1-\frac{\sqrt{s}\left(U_{2, O}-U_{2}\right)}{\left(v_{z}-U_{2}\right)}\right]\left(\frac{m_{O}}{2 \pi K T_{1}}\right)^{\frac{3}{2}} \exp \left[-\frac{m_{O}}{2 K s T_{1}}\left(U_{2}-v_{z}+\sqrt{s}\left(U_{2, O}-U_{2}\right)\right)^{2}\right]$

for $\quad \phi=\pi, \quad v_{z}<U_{2}-\sqrt{s}\left(U_{2, O}-U_{2}\right) ; \quad$ otherwise $f_{O^{+}, 2}\left(v_{z}\right) \equiv 0$. A cut for $v_{\|, 2}$ for the ring distribution function specified in Eq. (17) for the gyration clock angles from the range $(0, \pi)$ is shown in Fig. 3 (gray line).

In addition, the distribution function for the $\mathrm{O}$-atoms immediately after the passage from the upstream to the downstream side of the bow shock is not changed and thus is simply given by:

$$
\begin{aligned}
f_{O, 2}\left(v_{\perp, 2}, v_{\|, 2}\right) & =n_{O, 1}\left(\frac{m_{O}}{2 \pi K T_{1}}\right)^{3 / 2} \\
\cdot \exp & {\left[-\frac{m_{O}}{2 K T_{1}}\left(v^{2}+U_{1}^{2}-2 U_{1} v \cos \theta\right)\right] }
\end{aligned}
$$

where $\theta$ is the inclination of $\boldsymbol{v}\left(v_{\perp, 2}, v_{\|, 2}\right)$ to the bulk velocity vector $\boldsymbol{U}_{1}$, and for a cut along the stagnation line $(\theta=0)$ we have the unnormalized distribution function:

$$
\begin{aligned}
& f_{O, 2}\left(v_{z}\right)=\left(\frac{m_{O}}{2 \pi K T_{1}}\right)^{\frac{3}{2}} n_{O, 1} \\
& \cdot \exp \left[-\frac{m_{O}}{2 K T_{1}}\left(v_{z}-U_{1}\right)^{2}\right]
\end{aligned}
$$

\section{Results}

The distribution function of $\mathrm{O}^{+}$-ions immediately downstream of the bow shock strongly depends on the assumptions adopted for the process of shock transition. Due to an overshoot of velocity of the $\mathrm{O}^{+}$-ions immediately downstream, a ring distribution appears in the velocity space. This distribution function will be "picked up" by the magnetic field frozen in the proton plasma and convected towards the heliopause. Its $v_{z}$-cut in the velocity space (solar frame) along the inflow axis features two separate peaks (see Fig. 3). The separation of the peaks in the velocity space depends strongly on the intensity of the magnetic field, as illustrated in Fig. 3. While one of the peaks will always correspond to velocities directed sunward, the other one will either always be directed away from the Sun, as in the case of a mild MHD shock, or - in the case of an abrupt shock - will be partly or entirely (B-field dependent) directed sunward as well.
The $\mathrm{O}^{+}$-ions will exchange charge with the $\mathrm{H}$-atoms and get neutralized, producing a new population of $\mathrm{O}$-atoms with a signature inherited from the ring distribution of the parent $\mathrm{O}^{+}$-ions. At the same time, the original interstellar O-atoms will be exchanging charge with the protons within the interface, producing new $\mathrm{O}^{+}$-ions. The newly-created ions will be picked up by the magnetic field frozen in the interface flow, creating another ring population separate from the primary one, as shown in Fig. 4. Shown in this figure is the original population of the $\mathrm{O}^{+}$-ions, which (assuming no relaxation processes) will be convected with the bulk plasma flow towards the heliopause, suffering losses from charge exchange, and the new population of the $\mathrm{O}^{+}$-ions making another ring inside the original one and growing in number due to charge exchange induced implantations. Estimates of the charge exchange rate (see, e.g. Izmodenov et al., 2004) show that the density of the original $\mathrm{O}$-atom population should decrease at the heliopause to about 0.7 of the original content at the bow shock, with corresponding increase of the secondary $\mathrm{O}^{+}$-population.

The $\mathrm{O}^{+}$-ion distribution function shown in Fig. 3 allows to get a qualitative insight into a kind of signature imprinted in the shock transition upon the $\mathrm{O}$-atom velocity distribution function. The results obtained for the two cases considered, i.e. for an abrupt and mild MHD shocks, correspondingly with non-conservation and conservation of the magnetic moment of the $\mathrm{O}^{+}$-ions on the bow-shock passage, agree qualitatively (although quantitative differences do exist). The separation of maxima in the $v_{z}$-cut of the $\mathrm{O}^{+}$-ions velocity distribution function depends sensitively on the assumed magnetic field strength on the upstream side. For very weak upstream $B \simeq 0.1-0.5 \mu \mathrm{G}$ the shock is relatively strong, resulting in large $\mathrm{O}^{+}$-ion overhoot velocity and thus large separation of the two peaks in the $v_{z}$-coordinate. One of the peaks is then at positive (= antisunward) velocities, and transcharging of ions belonging to this peak will not create neutral O-atoms that could reach the inner heliosphere. On the other hand, for $B \simeq 2 \mu \mathrm{G}$ (which seems a more plausible case, see, e.g. Lallement et al., 2005 and Izmodenov et al., 2005) the shock becomes weak enough, i.e. the overshoot velocity small enough, to allow both peaks to appear at the 



Fig. 3. Ring distribution function (normalized) of $\mathrm{O}^{+}$-ions immediately downstream from the bow shock for the mild (black lines) and abrupt MHD bow shock (blue lines) for different values of magnetic field in the LIC, listed in the headers of the panels. Dashed line shows the corresponding normalized distribution function of neutral O-atoms immediately downstream of the shock.

negative (= sunward) side of the velocity distribution function (cf. Fig. 3). Thus in this case the $\mathrm{O}^{+}$-ions belonging to both peaks may, upon transcharging, give rise to O-atom fluxes that flow towards the inner heliosphere. In this way the population of $\mathrm{O}$-atoms created in the vicinity of the shock transition should in principle carry a two-peak signature of
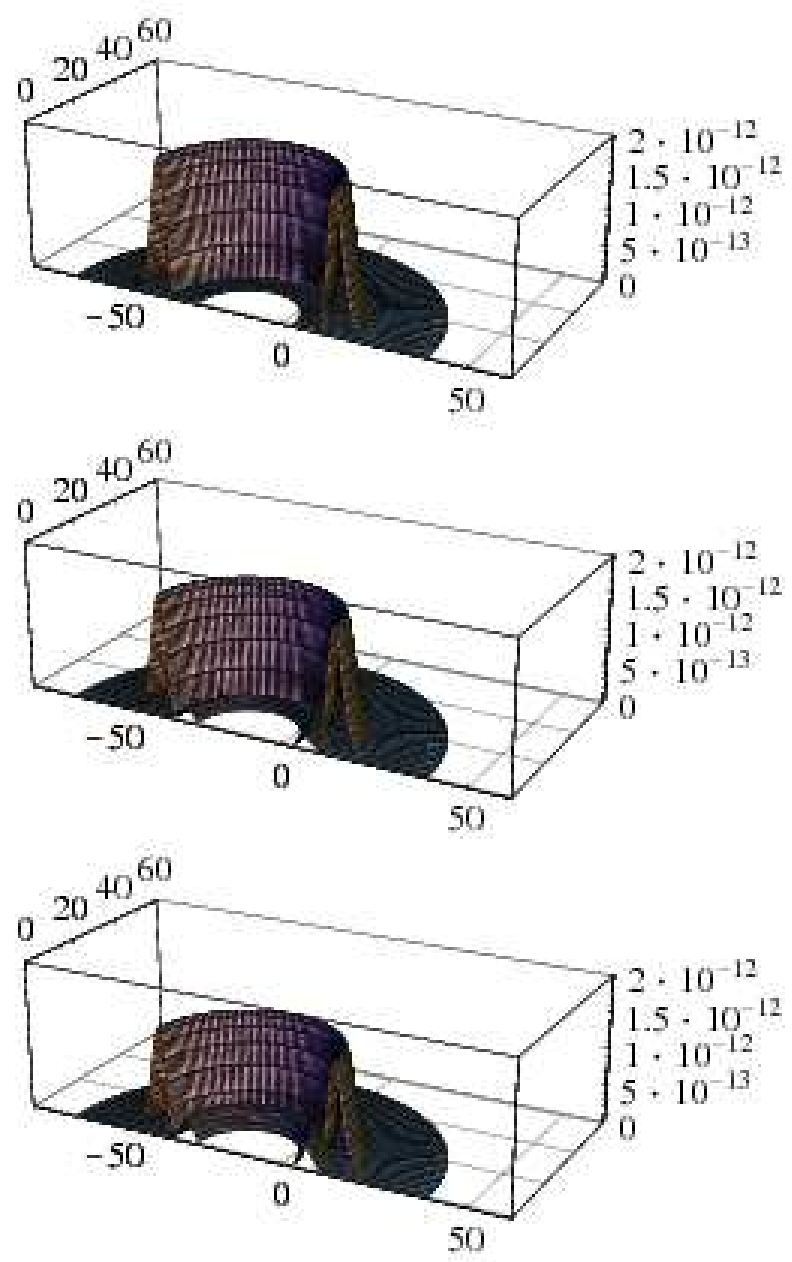

Fig. 4. The two-ring distribution function of $\mathrm{O}^{+}$-ions at three positions downstream from the bow shock. Immediately after the bow shock (upper panel) the secondary ring, arising due to the ionization of neutral oxygen atoms by charge exchange with neutral $\mathrm{H}$-atoms, is absent. Farther downstream, the secondary ring becomes visible (middle panel), growing with the increasing penetration into the outer heliosheath (lower panel). Click for high resolution figure.

the effective shock strength, i.e. of the magnitude of the upstream (= interstellar) magnetic field.

Relaxation processes, acting towards merging the charged population with the surrounding interface plasma, will reduce the visibility of these new populations. In particular, the ratio of numbers of neutral atoms created by the transcharging the two peaks at the $\mathrm{O}^{+}$-ion ring distribution emerging at the abrupt shock transition (blue curves in Fig. 3) may be very strongly affected by subsequent passage of the new $\mathrm{O}$-atoms through the heliospheric interface region. This results from comparing the efficiency of the production processes of O-atoms with the efficiency of the loss process for the two peaks of the 'blue' distributions in Fig. 3 (case with $B=2 \mu \mathrm{G}$ ). The production term will be proportional to $\alpha_{c x} \tau_{\text {eff }}$, where $\alpha_{c x}$ is the transcharge rate (in $\mathrm{cm}^{3} \mathrm{~s}^{-1}$ ) and $\tau_{\text {eff }}$ (in $\mathrm{s}$ ) is the lifetime of the double-humped distribution 
against all relaxation processes mentioned earlier. While $\alpha_{c x}$ depending on the collision frequency and charge exchange cross section may (moderately) differ for the high velocity $\left(v_{z} \simeq-26.5 \mathrm{~km} / \mathrm{s}\right)$ and low velocity $\left(v_{z} \simeq-5 \mathrm{~km} / \mathrm{s}\right)$ peaks, the lifetime $\tau_{\text {eff }}$ is obviously the same. Therefore the ratio of production of O-atoms by the two peaks should be of the order of the ratio of corresponding $\alpha_{c x}$ values.

In contrast, the destruction rate of the new O-atoms created by the two velocity peaks may differ drastically. In a first approximation, the loss rate $\alpha_{\text {loss }}\left(\mathrm{cm}^{3} \mathrm{~s}^{-1}\right)$ of the $\mathrm{O}$-atoms can be described as a combined effect of all loss processes like charge exchange, photo- and electron impact ionization, and scattering by elastic collisions. Therefore, the number $n_{O}$ of $\mathrm{O}$-atoms created in a given velocity peak will decrease according to

$$
\frac{d n_{O}}{d t}=-\alpha_{\text {loss }} t
$$

and the final result depends exponentially on the residence time $\tau_{\text {res }}$ of these atoms in the heliospheric interface layer. Therefore, as $\tau_{\text {res }}$ is larger by a factor of 5.3 for the slow peak $\left(v_{z} \simeq-5 \mathrm{~km} / \mathrm{s}\right.$, cf. Fig. 3) than for the fast peak $\left(v_{z} \simeq-26.5 \mathrm{~km} / \mathrm{s}\right)$, the suppression factor of the population of $\mathrm{O}$-atoms at the slow peak will (even for equal $\alpha_{\text {loss }}$ ) be $\exp (5.3) \simeq 200$ higher than at the fast peak. Therefore chances of detecting these imprints in the inner-heliospheric O-ENA distributions depend very sensitively - as illustrated by the two model cases discussed in Sects. 4.1 and $4.2-$ on the $\mathrm{O}^{+}$-ion behavior at the shock transition. We find that this result is one of the main conclusions of the paper and therefore we intend to proceed with a more detailed study of this effect in the future.

In principle, the evolution of the $\mathrm{O} / \mathrm{O}^{+}$-distribution functions downstream from the bow shock through the outer heliospheric interface has to be quantitatively followed with the help of corresponding Boltzmann-Vlasov equations, as already carried out for a similar problem of the description of the $\mathrm{H} / \mathrm{H}^{+}$-distribution functions by Fahr and Bzowski (2004a). In these equations, changes of the distribution functions due to the underlying changes with space coordinates of the magnetic field and of the plasma bulk velocity had to be quantitatively taken into account. Also the changes in time due to ion-atom collisions, to charge exchange collisions and to wave-particle interactions had to be correctly followed. This comprehensive evolutionary study is presently in progress, but will only be published in an upcoming paper.

Acknowledgements. M.B. and S.G. wish to acknowledge the hospitality and friendliness of the staff of the Institute of Astrophysics of the Bonn University (AIUB), where most of the work was carried out. This research was supported by the DFG/PAS Cooperative Project 436 POL 113/80/0 and by the Polish Ministry of Scientific Research and Information Technology Grant No 1P03D 00927. Edited by: K. Scherer

Reviewed by: I. V. Chashei and M. Scholer

\section{References}

Baranov, V. B. and Malama, Y. G.: Model of the solar wind interaction with the Local Interstellar Medium: numerical solution of self-consistent problem, J. Geophys. Res., 98, 15 157-15 163, 1993.

Baranov, V. B. and Malama, Y. G.: Effect of local interstellar medium fractional ionization on distant solar wind and interface region, Astron. Astrophys., 304, 14 755-14 761, 1995.

Bzowski, M., Fahr, H.-J., and Ruciński, D.: Ionisation induced heat flow in heliospheric hydrogen: virtues and flaws of hydrodynamic treatments, Astrophys. J., 544, 496-507, 2000.

Cameron, A. G. W.: A new table of abundances, in: Explosive Nucleosynthesis, edited by Schramm, D. N. and Arnett, W. D., 3, 1973.

Chambaud, G., Launay, J. M., Levy, B., Millie, P., Roueff, E., and Tran-Minh, F.: Charge Exchange and Fine Structure Excitation in O-H ${ }^{+}$Collisions, J. Phys., 13, 4205-4216, 1980.

Cox, D. P. and Helenius, L.: Flux-Tube Dynamics and a Model for the Origin of the Local Fluff, Astrophys. J., 583, 205-228, 2003.

Diver, D. A.: A plasma formulary for physics, technology, and astrophysics, Wiley - CVH, Berlin, Germany, 136-140, 2001.

Fahr, H.-J.: The multifluid character of the 'Baranov' interface, Astrophys. Space Sci., 274, 35-54, 2000.

Fahr, H.-J.: The charge-exchange induced coupling between plasma-gas counterflows in the heliosheath, Annales Geophys., 21, 1289-1294, 2003.

Fahr, H.-J. and Bzowski, M.: A semikinetc view on chargeexchange-induced perturbations of ion and atom distribution functions in the heliospheric interface, in: Proc. 3-rd Annual IGPP-UCR Astrophysics Conference: Physics of the Outer Heliosphere, edited by Forinski, V., Pogorelv, N. V., and Zank, G. P., AIP, 719, 161-181, 2004a.

Fahr, H.-J. and Bzowski, M.: A kinetic control on the heliopheric interface hydrodynamics of charge-exchanging fluids, Astron. Astrophys., 424, 263-278, 2004b.

Fahr, H.-J. and Lay, G.: Remote diagnostic of the heliospheric termination shock using neutralized post-shock pick-up ions as messengers, Astron. Astrophys., 356, 327-334, 2000.

Fahr, H. J. and Ripken, H. W.: The physics of the heliospheric interface and its implications for LISM diagnostics, Astron. Astrophys., 139, 551-554, 1984.

Fahr, H.-J., Osterbart, R., and Ruciński, D.: The effect of the heliospheric filtration on the distant Lyman-Alpha glow and the pickup proton fluxes, Astron. Astrophys., 274, 612-620, 1993.

Fahr, H.-J., Kausch, T., and Scherer, H.: A 5-fluid hydrodynamic approach to model the solar system - interstellar medium interaction, Astron. Astrophys., 357, 268-282, 2000.

Frisch, P. C.: Boundary conditions of the heliosphere, J. Geophys. Res., 108, 8036, 10.1029/2003JA009 909, 2003.

Frisch, P. C.: The Solar Galactic Environment, in: AIP Conf. Proc. 719: Physics of the Outer Heliosphere, 404-411, 2004.

Izmodenov, V. V., Malama, Y., Gloeckler, G., and Geiss, J.: Filtration of interstellar $\mathrm{H}, \mathrm{O}, \mathrm{N}$ atoms through the heliospheric interface: Inferences on local interstellar abundances of the elements, Astron. Astrophys., 414, L29-L32, 2004.

Izmodenov, V. V., Alexashov, S., and Myasnikov, A.: Direction of the interstellar $\mathrm{H}$ atom flow in the heliosphere: role of the interstellar magnetic field, Astron. Astrophys., 437, L35-L38, 2005.

Izmodenov, V. V., Lallement, R., and Malama, Y. G.: Interstellar neutral oxygen in a two-shocks heliosphere, Astron. Astrophys., 317, 193-202, 1997. 
Kingdon, J. B. and Ferland, G. J.: Rate Coefficients for Charge Transfer between Hydrogen and the First 30 Elements, Astrophys. J.Suppl, 106, 205-211, 1996.

Lallement, R., Quémerais, E., Bertaux, J. L., Ferron, S., Koutroumpa, D., and Pellinen, R.: Deflection of the Interstellar Neutral Hydrogen Flow Across the Heliospheric Interface, Science, 307, 1447-1449, 2005.

Lee, L. C., Wu, C. S., and Hu, X. W.: Increase of ion kinetic temperature across a collisionless shock. I - A new mechanism, Geophys. Res. Lett., 13, 209-212, 1986.

Maher, L. J. and Tinsley, B. A.: Atomic hydrogen escape rate due to charge exchange with hot plasmaspheric ions, J. Geophys. Res., 82, 689-695, 1977.

Malama, Y.: Monte-Carlo simulation of neutral atoms trajectories in the solar system, Astrophys.Space.Sci., 176, 21-46, 1991.

Mancuso, S., Raymond, J. C., Kohl, J., Ko, Y.-K., Uzzo, M., and Wu, R.: UVCS/SOHO observations of a CME-driven shock: Consequences on ion heating mechanisms behind a coronal shock, Astron. Astrophys., 383, 267-274, 2002.

McNutt, R. L., Lyon, J., and Goodrich, C. C.: Simulation of the heliosphere: model, J. Geophys. Res., 103, 1905-1912, 1998.
Oliveira, C., Dupuis, J., Chayer, P., and Moos, H. W.: O/H in the Local Bubble, Astrophys. J., 587, 235-255, 2005.

Osterbart, R. and Fahr, H.-J.: A Boltzmann-kinetic approach to describe entrance of neutral interstellar hydrogen into the heliosphere, Astron. Astrophys., 264, 260-269, 1992.

Ripken, H. W. and Fahr, H.-J.: Modification of the local gas properties in the heliospheric interface, Astron. Astrophys., 122, 181-192, 1983.

Stebbings, R. F., Smith, A. C. H., and Ehrhardt, H.: Charge Transfer between Oxygen Atoms and $\mathrm{O}^{+}$and $\mathrm{H}^{+}$Ions, J. Geophys. Res., 69, 2349-2355, 1964.

Witte, M.: Kinetic parameters of interstellar neutral helium. Review of results obtained during one solar cycle with the Ulysses/GAS instrument, Astron. Astrophys., 426, 835-844, 2004.

Witte, M., Banaszkiewicz, M., Rosenbauer, H., and McMullin, D.: Kinetic parameters of interstellar neutral helium: updated results from the ULYSSES/GAS-instrument, Adv. Space Res., 34, 61-65, 2004.

Zank, G. P.: Interaction of the solar wind with the Local Interstellar Medium: a theoretical perspective, Space Sci. Rev., 89, 413-688, 1999. 